\title{
Postpartum Acute Renal Failure
}

National Cancer Institute

\section{Source}

National Cancer Institute. Postpartum Acute Renal Failure. NCI Thesaurus. Code

C114388.

Renal failure that occurs postpartum due to any partum problem (hemorrhage, sepsis, preeclampsia). 